BIOLOGICAL CRYSTALLOGRAPHY

ISSN 1399-0047

Received 19 September 2014

Accepted 4 March 2015

Edited by G. J. Kleywegt, EMBL-EBI, Hinxton, England

Keywords: serial crystallography; hit finding; raster scanning; background subtraction; radiation damage.

PDB references: hen egg-white lysozyme, $4 \mathrm{wg} 1 ; 4 \mathrm{wg} 7 ; 4 \mathrm{wl} 6 ; 4 \mathrm{wl} 7$

Supporting information: this article has supporting information at journals.iucr.org/d

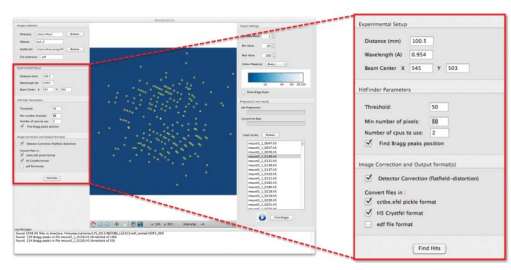

OPEN $\odot$ ACCESS

\section{Raster-scanning serial protein crystallography using micro- and nano-focused synchrotron beams}

\author{
Nicolas Coquelle, ${ }^{\mathrm{a}, \mathrm{b}, \mathrm{c}}$ Aaron S. Brewster, ${ }^{\mathrm{d}}$ Ulrike Kapp, ${ }^{\mathrm{e}}$ Anastasya Shilova, \\ Britta Weinhausen, ${ }^{e}$ Manfred Burghammer ${ }^{\mathrm{e}, \mathrm{f}_{*}}$ and Jacques-Philippe Colletier ${ }^{\mathrm{a}, \mathrm{b}, \mathrm{c} *}$ \\ ${ }^{\mathbf{a}}$ Université Grenoble Alpes, IBS, 38044 Grenoble, France, ${ }^{\mathbf{b}} \mathrm{CNRS}$, IBS, 38044 Grenoble, France, ${ }^{\mathbf{c}} \mathrm{CEA}$, IBS, \\ 38044 Grenoble, France, ${ }^{\mathbf{d} P h y s i c a l}$ Biosciences Division, Lawrence Berkeley National Laboratory, Berkeley, CA 94720, \\ USA, 'european Synchrotron Radiation Facility, BP 220, 38043 Grenoble, France, and 'Department of Analytical \\ Chemistry, Ghent University, Ghent B-9000, Belgium. *Correspondence e-mail: burgham@esrf.fr, colletier@ibs.fr
}

High-resolution structural information was obtained from lysozyme microcrystals $(20 \mu \mathrm{m}$ in the largest dimension) using raster-scanning serial protein crystallography on micro- and nano-focused beamlines at the ESRF. Data were collected at room temperature (RT) from crystals sandwiched between two silicon nitride wafers, thereby preventing their drying, while limiting background scattering and sample consumption. In order to identify crystal hits, new multi-processing and GUI-driven Python-based pre-analysis software was developed, named NanoPeakCell, that was able to read data from a variety of crystallographic image formats. Further data processing was carried out using CrystFEL, and the resultant structures were refined to $1.7 \AA$ resolution. The data demonstrate the feasibility of RT raster-scanning serial micro- and nanoprotein crystallography at synchrotrons and validate it as an alternative approach for the collection of high-resolution structural data from micro-sized crystals. Advantages of the proposed approach are its thriftiness, its handlingfree nature, the reduced amount of sample required, the adjustable hit rate, the high indexing rate and the minimization of background scattering.

\section{Introduction}

The advent of X-ray free-electron lasers (XFELs) has driven the emergence of serial protein crystallography (Chapman $e t$ al., 2011). In this novel approach, data are collected at room temperature (RT) from tens to hundreds of thousands of 'still' crystals, all of which are in different, more or less random, orientations. In contrast, the vast majority of the nearly 94000 $\mathrm{X}$-ray structures deposited in the PDB were obtained from data collected at cryogenic temperature (generally $100 \mathrm{~K}$ ) from a single crystal rotated in an X-ray beam. Compared with RT, such low temperatures reduce by $\sim 100$-fold the radiation damage that accumulates in crystals during data collection and degrades the structural information (Nave \& Garman, 2005). The price to pay is, however, that of reduced insight into protein conformational heterogeneity and dynamics (Motlagh et al., 2014; Fraser et al., 2011). Kinetic crystallography approaches have been developed to overcome the static nature of cryo-crystallographic structures, but none are universally applicable (Bourgeois \& Royant, 2005). Arguably, methodologies to collect high-resolution X-ray data at RT are needed to better understand the interplay between structure and dynamics and how they synergistically give rise to protein function (Weik \& Colletier, 2010).

XFELs deliver roughly as many photons in a 5-50 fs pulse as an insertion-device beamline at a third-generation 
synchrotron does in a second. Such short exposures allow the collection of RT diffraction data before crystals are destroyed by the intense X-ray pulse (Chapman et al., 2011; Neutze et al., 2000). At XFELs, samples are commonly streamed across the $\mathrm{X}$-ray beam by virtue of an injector. Because crystals virtually stand still (no oscillation or tumbling) during the time-lapse of their exposure to the X-rays, only a thin slice of the reciprocal space is recorded for each. The production of a full data set therefore requires the collection of patterns from many thousands of crystals in an approach termed 'serial femtosecond crystallography' (SFX; Chapman et al., 2011). Over the last four years, this approach has demonstrated its power, notably by enabling high-resolution data to be collected from submicrometre-sized crystals (Boutet et al., 2012; Koopmann et al., 2012; Redecke et al., 2013; Sawaya et al., 2014; Stevenson et al., 2014). XFELs also hold the promise of soon allowing subpicosecond time-resolved structural studies, which are technically unfeasible at third-generation synchrotron radiation (SR) sources (Neutze, 2014). These SFX developments have been accompanied by active software development in order to contend both with the thinness of the three-dimensional reciprocal slice collected from still crystals and with the colossal amount of data produced during experiments.

In SFX, samples are often presented to the X-ray beam by means of gas dynamic virtual nozzle (GDVN)-type injectors. GDVNs can be used to compress an aqueous crystal stream, thereby generating a liquid jet (DePonte et al., 2009). They can also be used to push a crystal-containing lipid cubic phase (LCP) across the X-ray beam (Weierstall et al., 2014). Liquid GDVN injectors require monumental amounts of sample (hundreds of milligrams), and thus are only suited for targets whose production, purification and crystallization are straightforward and streamlined. LCP injectors are slower and therefore comparatively economical $(<1 \mathrm{mg})$, but the range of protein crystals that are amenable to embedding in LCP is as yet unknown. Also, resuspending crystals into LCP can be difficult and LCP injectors can suffer from drying and phase transitions. A possible alternative is to embed crystals into mineral grease and to use an LCP injector to flow them across the X-ray beam (Sugahara et al., 2015). A nanoflow electrospinning injector has been described in which high voltages are used instead of high pressures to flow crystals across the X-ray beam (Sierra et al., 2012). Use of this technology reduces the amount of sample required down to hundreds of micrograms (Sierra et al., 2012), but requires that crystals are grown in, or are transferable into, a viscous mother liquor. A system based on acoustic droplet ejection has also been described (Soares et al., 2011). Common to all these approaches is that crystals are literally drowned in solvent, leading to high and fluctuating background scattering that complicates data processing. Alternate sample-handling methods are therefore amongst the most critical developments required for the further emergence of SFX. Recently, a method for the collection of data from crystalline samples dried onto fixed targets has been presented (Hunter et al., 2014), as well as a goniometer-based approach to SFX (Cohen et al., 2014).
The worldwide availability of SR sources raises the possibility that some of the experiments currently performed at XFELs could be implemented at synchrotrons. As already mentioned, the excitement elicited by XFELs has led scientific programmers to challenge themselves with the handling and processing of millions of diffractions patterns, making it now possible to process serial crystallographic data regardless of the X-ray sources they are collected at. To date, at least two major software suites exist: CrystFEL (White et al., 2012) and cctbx.xfel (Sauter et al., 2013). Subtleties in the processing approaches of the two software suites have recently been discussed (Sawaya et al., 2014).

In early 2014, a pioneering study reported serial synchrotron crystallography (SSX) experiments on Trypanosoma brucei cathepsin B crystals (CatB) mounted in a nylon loop at $110 \mathrm{~K}$ (Gati et al., 2014). Data were collected using a combination of raster scanning and oscillation, whereby evenly spaced $(5 \mu \mathrm{m})$ volumes of the nylon-loop content were illuminated by a $4 \times 5 \mu \mathrm{m}$ beam while oscillating the loop by $90^{\circ}$ over the complete scan. Hence, a three-dimensional wedge, rather than a thin slice of the reciprocal lattice, was collected at each exposure. More recently, another consortium reported serial synchrotron crystallography data collected at RT from lysozyme crystals flowed across a $6 \times 9 \mu \mathrm{m} X$-ray beam inside a capillary (Stellato et al., 2014). Using this new approach, the authors were able to collect $\sim 1.6 \times 10^{6}$ frames in about $17 \mathrm{~h}$, of which $\sim 40 \times 10^{3}$ were successfully indexed. A total of $2.5 \mathrm{ml}$ crystal slurry was consumed, corresponding to $250 \mathrm{mg}$ of protein. The data proved to be of excellent quality [as judged from statistical indicators such as $R_{\text {split }},\langle I / \sigma(I)\rangle, \mathrm{CC}_{1 / 2}$ and redundancy], producing a $2.1 \AA$ resolution structure comparable to that obtained by the collection of oscillation data at a cryogenic temperature. Two compelling advantages of this approach are that (i) pseudo-oscillation data can be collected from crystals rolling inside the capillary during the exposure time and (ii) frames can be acquired in a shutterless fashion, thus theoretically reducing the exposure time to the limit of detector readout. Among the remaining issues are, however, the amount of sample consumed and the overall success rate. A more economical approach is thus still required for proteins whose production and crystallization cannot reach a manufacturing scale.

In the present study, we report an alternative serial synchrotron crystallography approach allowing the collection of data at RT and in a pure raster-scanning mode; that is, without oscillating the crystals. For this proof of concept, we used tetragonal microcrystals of chicken hen egg-white lysozyme so as to allow a direct comparison of our data with those recently produced by SFX (Boutet et al., 2012) and SSX (Stellato et al., 2014). To determine the minimal beam size to perform such experiments at SR facilities, data were collected using either a micro-focused or a nano-focused beam. Samples were presented to the $\mathrm{X}$-ray beam in a silicon nitride $\left(\mathrm{Si}_{3} \mathrm{~N}_{4}\right)$ sandwich, thence avoiding the drying of samples while limiting background scattering and sample consumption. Identification of crystal hits, background subtraction and peak searching were performed using NanoPeakCell, a new multi-processing 
and GUI-driven Python-based pre-analysis software. Further processing of the data was carried out with CrystFEL, and structures collected using the micro- and nano-focused beams were refined to $1.7 \AA$ resolution. We propose the use of RT raster-scanning serial protein crystallography using SR microand nano-focused beams as an alternative approach for the collection of high-resolution structural data from micrometresized crystals. The proposed method is thrifty and virtually handling-free, and so can be used on fragile and scarce microcrystalline samples.

\section{Materials and methods}

\subsection{Description of the ESRF Microfocus Beamline (ID13)}

ID13 is a versatile beamline that provides ESRF users with intense micro-focused $(0.3-2.5 \mu \mathrm{m}$, micro-branch, EH2) and nano-focused (100-500 nm; nano-branch, EH3) beams. The focusing optics used to generate the micro-beam are Be-based compound refractive lenses (CRL) consisting of 56 single lenses. Each lens has a curvature radius of $50 \mu \mathrm{m}$ in the apex. For the nano-beam, Si-based linear compound refractive lenses in crossed geometry (so-called NFL lenses; Schroer et al., 2005) are used.

At the micro-branch of ID13, sample centring and positioning are performed with translation stages (Micos, Germany), with $200 \mathrm{~nm}$ incremental steps in the $x / y$ directions (HPS-170) and an overall travel range of $160 \mathrm{~mm}$, and incremental steps of $100 \mathrm{~nm}$ in the vertical direction (UPL-160) with a travel range of $25 \mathrm{~mm}$. In the nano-branch, the sample centring and scanning are operated via a P622K075 piezo $z$ stage (vertical direction) mounted on a P622K074 piezo $x y$ stage. The minimum incremental movement as mounted on the nano-setup of ID13 is $10 \mathrm{~nm}$ in the vertical direction and $20 \mathrm{~nm}$ in the two horizontal directions. This arrangement of piezo stages is mounted on an M-810 miniature hexapod. The piezo stages and the hexapod were manufactured by PhysikInstrumente (PI GmbH, Karlsruhe, Germany). The hexapod and the piezo stage can translate in the three spatial dimensions over distances of about $10 \mathrm{~mm}$ and $250 \mu \mathrm{m}$, respectively.

Diffraction data were recorded using an ESRF-built Frelon $4 \mathrm{M} \mathrm{CCD}$ area detector with $2048 \times 2048$ pixels and $50 \mu \mathrm{m}$ pixel size (Coan et al., 2006) in the $2 \times$ binning mode $(1024 \times$ 1024 pixels and $100 \mu \mathrm{m}$ pixel size; $\sim 0.35 \mathrm{~s}$ readout time). Data

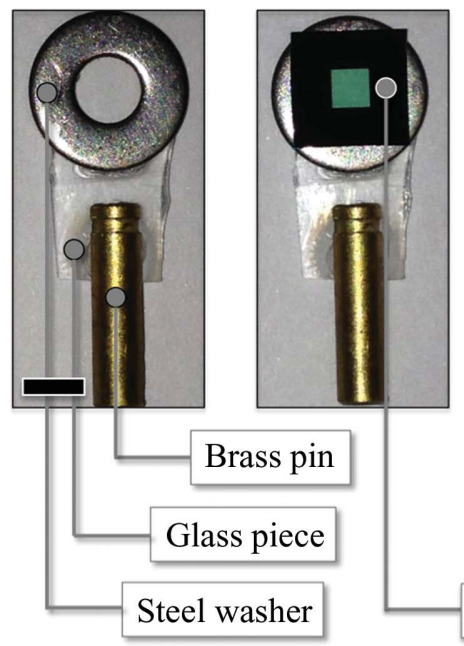

(a)

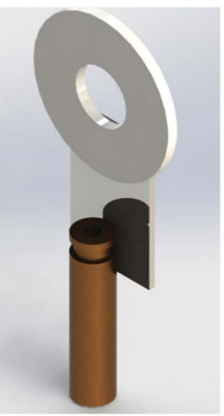

Figure 1

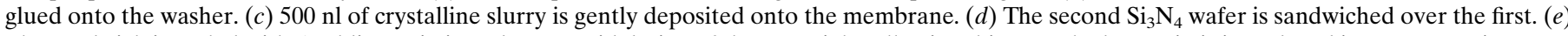

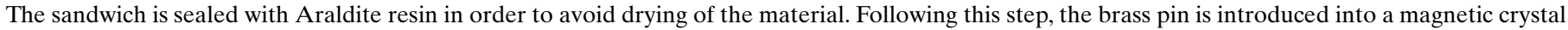
mount. $(f)$ Three-dimensional rendering of steps $(a)-(e)$. $(g, h)$ Overview $(g)$ and close-up view $(h)$ of the lysozyme crystals in the $\mathrm{Si}_{3} \mathrm{~N}_{4}$ sandwich. 
were collected in raster scanning mode, i.e. by translating samples through the focus in two directions perpendicular to the incident beam.

\subsection{Sample preparation and data collection}

Tetragonal $\left(P 4_{3} 2_{1} 2\right)$ micro-crystals $(20 \times 20 \times 20 \mu \mathrm{m})$ of hen egg-white lysozyme (HEWL) were grown using the vapour-diffusion method (Lomb et al., 2011) and directly pipetted from their drops into an Eppendorf tube. Gentle centrifugation for $300 \mathrm{~s}\left(6000 \mathrm{rev} \mathrm{min}^{-1}\right)$ settled the crystals at the bottom of the tube.

$500 \mathrm{nl}$ of the crystalline precipitate were pipetted onto a $500 \mathrm{~nm}$ thick $\mathrm{Si}_{3} \mathrm{~N}_{4}$ wafer (frame thickness $200 \mu \mathrm{m}$ ) before laying another $\mathrm{Si}_{3} \mathrm{~N}_{4}$ wafer (of the same thickness) onto it in a back-to-back fashion (Fig. 1). $\mathrm{Si}_{3} \mathrm{~N}_{4}$ wafers were from Silson, Northampton, England (http://www.silson.com). Sandwiching the $\mathrm{Si}_{3} \mathrm{~N}_{4}$ wafers back to back was essential to prevent crystals from slithering down during the diffraction experiment. The $\mathrm{Si}_{3} \mathrm{~N}_{4}$ sandwich was placed over a steel washer, glued onto it using SuperGlue and the sandwich border was hermetized using Araldite resin (Fig. 1a). The washer was glued onto a small glass piece, itself glued onto a brass pin that was introduced into an adjustable magnetic crystal mount. The usable $\mathrm{Si}_{3} \mathrm{~N}_{4}$ surface was $2.5 \times 2.5 \mathrm{~mm}$. For presentation to the X-ray beam, the mounts were either placed onto an $x y z$ translation table (micro-focused beam; ESRF ID13-EH2) or onto a miniature hexapod (nano-focused beam; ESRF ID13-EH3).

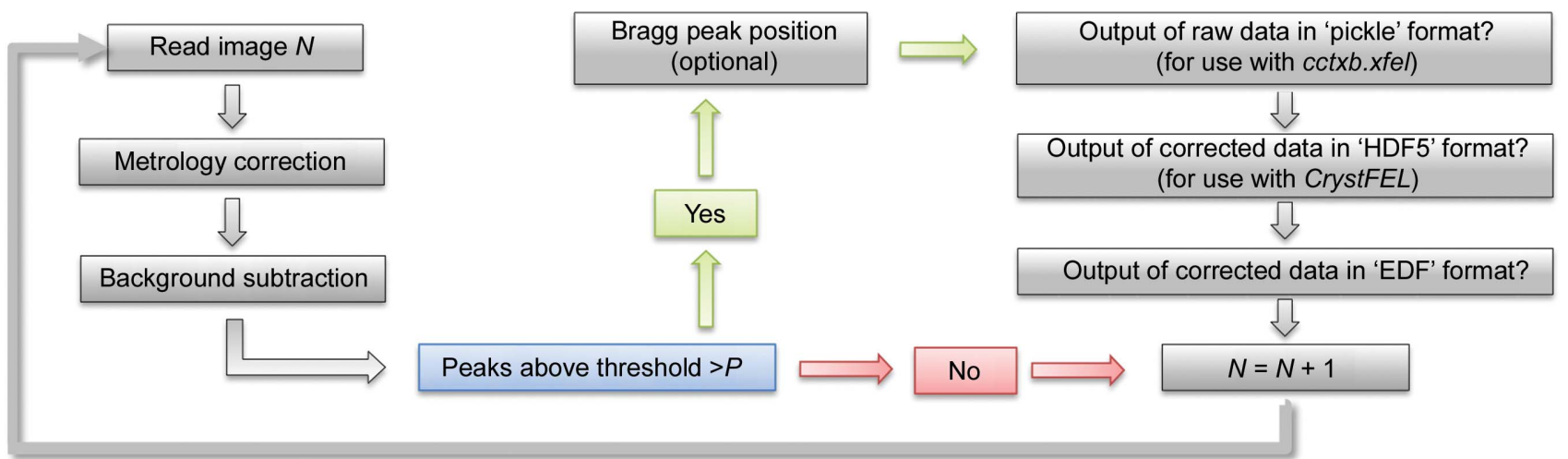

(a)

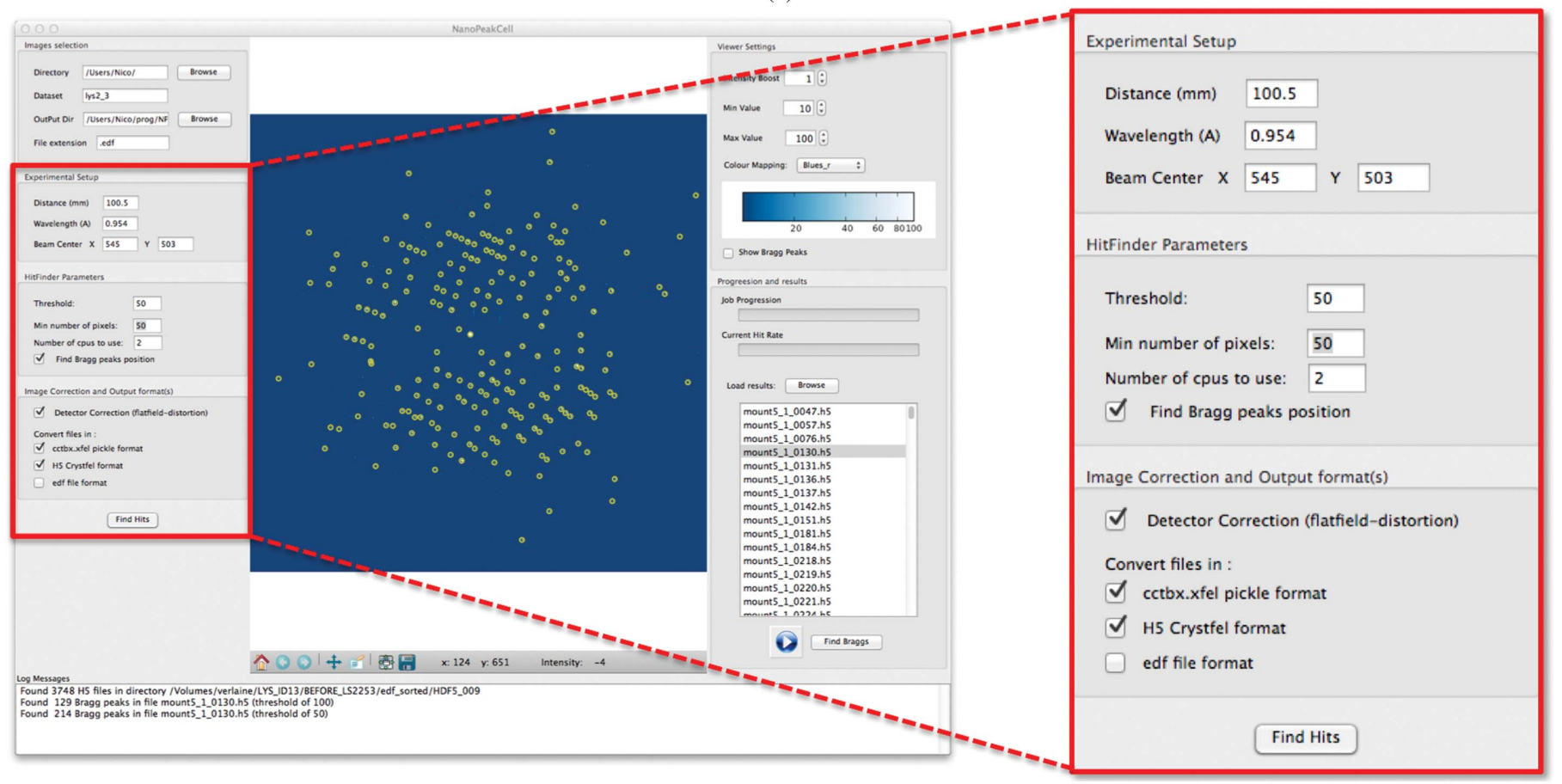

(b)

Figure 2

The new NanoPeakCell pre-analysis software for serial crystallography. (a) Workflow of the actions performed by NanoPeakCell. (b) Overview of the graphical user interface (GUI) of NanoPeakCell. On the left, the user can define important experimental parameters, as well as parameter values used during the hit-finding and peak-finding procedures (red inset). On the bottom left, the user can select the correction(s) to be performed and the output format(s) for saving hits (red inset). Each hit is displayed in the central panel, with yellow circles around Bragg peaks. NanoPeakCell can also be used as a data-visualization program. The right panel is dedicated to display adjustments and allows results from previous runs of NanoPeakCell to be reloaded for inspection. Users can also use NanoPeakCell to view raw data in any of the supported formats. 
Table 1

Data-collection parameters.

\begin{tabular}{|c|c|c|}
\hline Parameter & Micro & Nano \\
\hline $\begin{array}{l}\text { Beam size }(\text { vertical } \times \text { horizontal }) \\
\quad(\mathrm{FWHM})(\mu \mathrm{m})\end{array}$ & $1.5 \times 2.5$ & $0.150 \times 0.180$ \\
\hline Beam divergence (mrad) & 1.0 & 0.3 \\
\hline Wavelength $(\AA)$ & 0.954 & 0.832 \\
\hline Beam fluence (photons s ${ }^{-1}$ ) & $1.00 \times 10^{11}$ & $1.70 \times 10^{10}$ \\
\hline Flux density (photons $\mathrm{s}^{-1} \mathrm{~mm}^{-2}$ ) & $2.67 \times 10^{16}$ & $6.48 \times 10^{17}$ \\
\hline Brilliance (photons s${ }^{-1} \mathrm{~mm}^{-2} \mathrm{mrad}^{-2}$ ) & $2.67 \times 10^{22}$ & $6.09 \times 10^{24}$ \\
\hline Bandwidth $(\AA)$ & $<1.00 \times 10^{-4}$ & $<1.00 \times 10^{-4}$ \\
\hline Exposure time per frame (s) & 0.2 & 0.1 \\
\hline Dose per crystal $\uparrow(\mathrm{MGy})$ & 3.2 & 29.1 \\
\hline Dose rate $\left(\mathrm{MGy} \mathrm{s}^{-1}\right)$ & 16 & 291 \\
\hline
\end{tabular}

$\dagger$ As calculated using RADDOSE (Paithankar et al., 2009).

Data sets collected using the micro- and nano-focused beams are referred to as 'micro' and 'nano', respectively. Data collection was segmented into scans of 41 (horizontal, fast scanning) by 41 (vertical, slow scanning) steps, each started at a remote location on the wafer. The track length of photoelectrons from $1 \AA$ wavelength X-rays in water and protein has been estimated to be $\sim 3 \mu \mathrm{m}$ (Cole, 1969). Within the same scan, X-ray shots were accordingly spaced by $10(>2.5+3)$ and $5(>0.2+3) \mu \mathrm{m}$ for the micro (beam size $1.5 \times 2.5 \mu \mathrm{m})$ and nano (beam size $0.15 \times 0.18 \mu \mathrm{m}$ ) data sets, respectively (Table 1).

Exposure times were 0.2 and $0.1 \mathrm{~s}$ per shot for the micro and nano data sets, respectively. The flux densities of the microand nano-focused beams were $2.67 \times 10^{16}$ and $6.48 \times$ $10^{17}$ photons s${ }^{-1} \mathrm{~mm}^{-2}$, respectively, while the calculated doses per image (and thus the average doses of the data set) were 3.2 and 29.1 MGy, respectively (Table 1). The micro and nano data sets consist of $\sim 41$ and $\sim 84$ scans (69 319 and 139985 frames), corresponding to $\sim 9$ and $20 \mathrm{~h}$ of data collection, respectively. The rate of data acquisition was limited by the readout time of the detector.

\subsection{Data sorting and processing}

Regardless of whether the data are collected at an XFEL or at an SR source, a major challenge in serial crystallography is hit finding. Also, background scattering must be subtracted to permit an accurate measurement of the diffracted intensities (Boutet et al., 2012). Data correction must, however, be performed cautiously. Indeed, in a given volume crystals and solvent are mutually exclusive, so the amount of background to be subtracted needs to be scaled on a per-pattern basis. Specific to the use of a Frelon CCD camera, raw images must

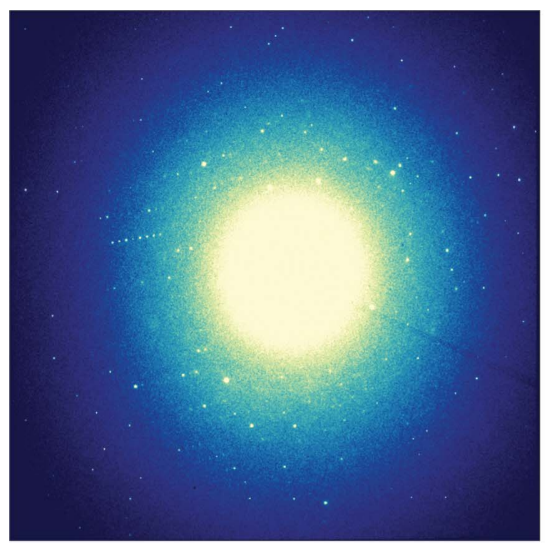

(a)

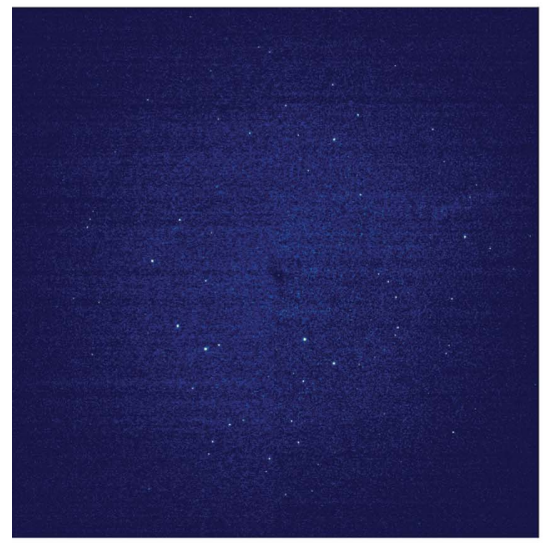

(d)

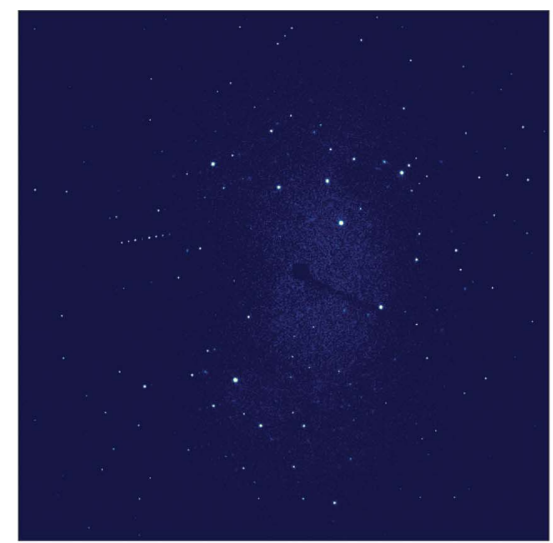

(b)

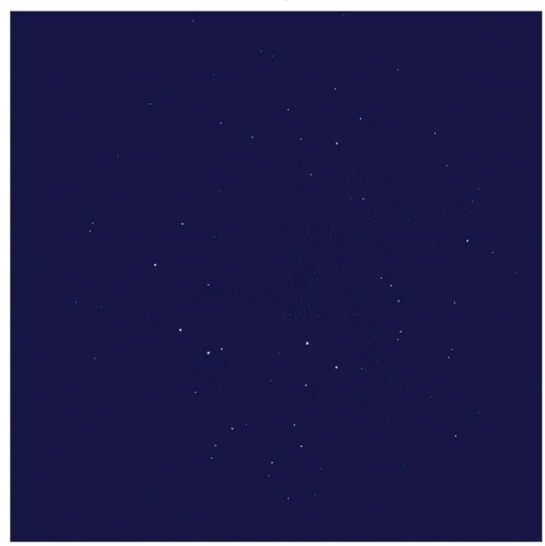

(e)

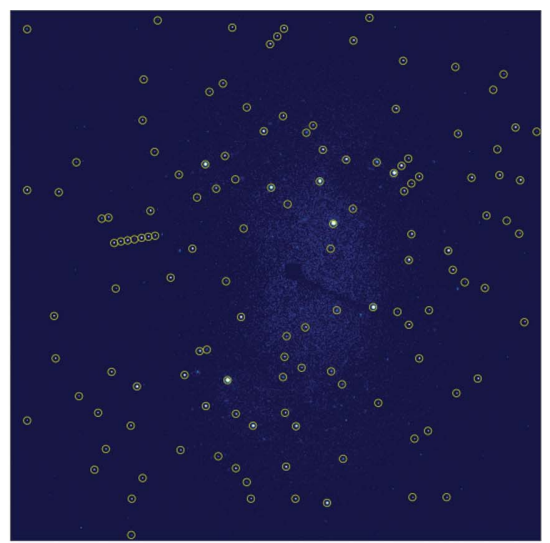

(c)

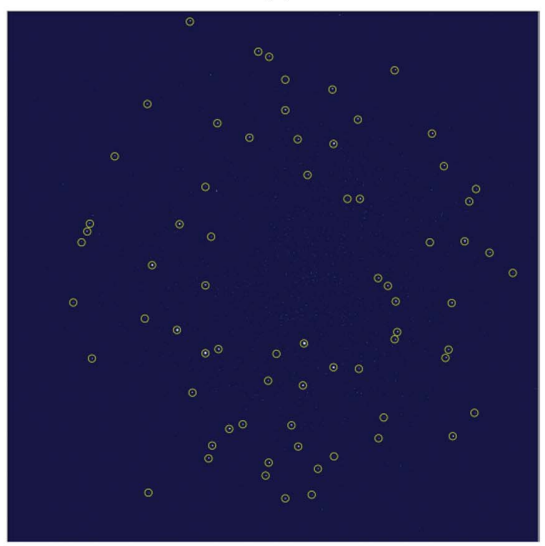

$(f)$

Figure 3

Data correction and finding Bragg peaks. $(a),(b)$ and $(c)$ show the same diffraction pattern from the micro data set before $(a)$ and after $(b, c)$ background subtraction. $(c)$ shows the Bragg peaks found by NanoPeakCell in this diffraction pattern. Likewise, $(d),(e)$ and $(f)$ show the same diffraction pattern from the nano data set before $(d)$ and after $(e, f)$ background subtraction. The Bragg peaks found by NanoPeakCell are highlighted in $(f)$ (yellow circles). 
Table 2

Crystallographic refinement statistics.

Values in parentheses are for the highest resolution shell.

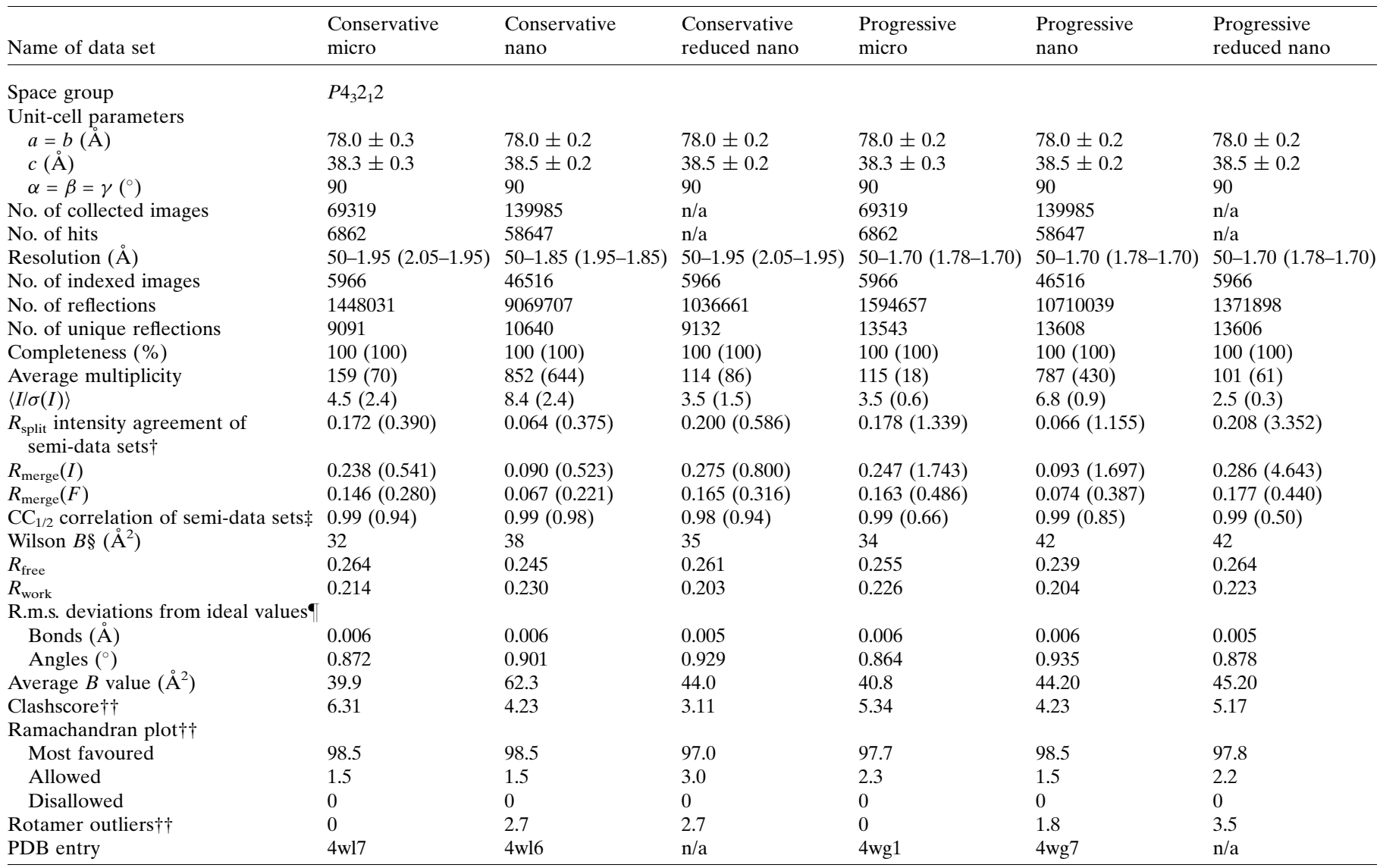

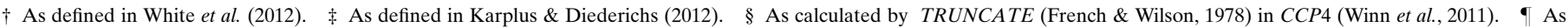
defined in Engh \& Huber (1991). †† As calculated by MolProbity (Chen et al., 2010).

also be corrected for distortion and a flat-field correction applied (metrology correction). Together, these requirements prompted us to undertake the coding of a multi-processing, GUI-driven Python-based hit finder and corrector, which we named NanoPeakCell. The flowchart of actions performed by NanoPeakCell is depicted in Fig. 2(a) and includes conversion of images (the supported input formats include EDF, SMV, MCCD, CBF, TIFF, HDF5, SACLA-HDF5 and LCLS-XTC) into a suitable format for data processing with CrystFEL (HDF5 format) and/or cctbx.xfel ('pickle' format), as well as the generation of the parameter files and scripts needed to run CrystFEL in a user-friendly fashion (Fig. 2b). Of note, NanoPeakCell not only selects hits (i.e frames with actual diffraction spots) on the basis of a single pixel being higher than a given threshold, but also checks whether a sufficient (user-provided) number of such pixels are present to allow further indexing. To allow determination of the effective resolution limit of the serial data set, NanoPeakCell additionally performs a maximum projection of selected hits on the fly (see Supplementary Fig. S1). Finally, NanoPeakCell performs Bragg-peak finding using a local maximum algorithm and outputs the peak list in a supplementary data set of the HDF5 file, if the user selects for this output format, or in a text file otherwise. This can allow the rapid elimination of both blank and low-resolution images. Similar indexing rates were obtained when using the peak-finding function implemented in CrystFEL and that implemented in NanoPeakCell. Using the latter, however, avoids spending time optimizing the peakdetection parameters in CrystFEL. Accordingly, in this work, Bragg-peak finding was performed using NanoPeakCell and peak locations were fed into CrystFEL (using the --peaks $=$ hdf 5 option in the indexamajig module) for indexing (Kirian et al., 2011; White et al., 2012, 2013). NanoPeakCell is installed on the ESRF ID13 micro-focused (ID13-EH2) and nano-focused beamlines (ID13-EH3), where it can be controlled either through the GUI or from the command line, and its development is continuing. Typical raw and corrected images from the micro and nano data sets are shown in Fig. 3. Others details concerning NanoPeakCell and its use at various facilities (including ESRF, LCLS and SACLA) will be published elsewhere.

Data were processed using CrystFEL (Kirian et al., 2011; White et al., 2012, 2013). CrystFEL indexing relied on MOSFLM (Powell et al., 2013), Dirax (Duisenberg, 1992) and $X D S$ (Kabsch, 2010). Bragg-peak integration was performed using the 'rings-nocen' method in CrystFEL, with concentric 
rings of five, seven and nine pixels to determine the peak, buffer and background regions, respectively. The radius of the three-dimensional intensity profile was $5 \times 10^{-4} \AA^{-1}$. The divergences of the micro- and nano-focused beams were determined experimentally (Table 1). Merging of integrated intensities was performed using Monte Carlo integration in CrystFEL (Kirian et al., 2011; Table 2).

The initial data processing was performed using conservative $\langle I / \sigma(I)\rangle(>2)$ and $R_{\text {split }}(\sim 50 \%)$ criteria to determine the highest resolution shells. Based on these criteria, the micro and nano data sets were integrated to 1.95 and $1.85 \AA$ resolution, respectively (Table 2). These two data sets, and their corresponding structures, are referred to as 'conservative micro' and 'conservative nano'. The statistics of the conservative data sets, shown in Table 2, are of excellent quality, as judged by $R_{\text {split }}, \mathrm{CC}_{1 / 2}$ and Wilson $B$ factor. Supplementary Fig. S2 shows the convergence of data-quality indicators such as $R_{\text {split }}$ and $\mathrm{CC}_{1 / 2}$ as a function of resolution and the number of images. It indicates that in both data sets the $\mathrm{CC}_{1 / 2}$ values at $1.7 \AA$ resolution remain well above the 0.135 limit advised by Karplus \& Diederichs (2012). The presence of diffraction signal up to this resolution in both data sets is also visible in the azimuthal integrations of the maximum projections of the indexed patterns (Supplementary Fig. S1). Thus, we performed a more progressive integration, in which $\mathrm{CC}_{1 / 2}$ and iterative pair refinement (Karplus \& Diederichs, 2012) were used instead of strict $\langle I / \sigma(I)\rangle$ and $R_{\text {split }}$ criteria to determine the high-resolution cutoffs. These two $1.7 \AA$ resolution data sets and their corresponding structures are referred to as 'progressive micro' and 'progressive nano'. The only difference between the progressive nano (or progressive micro) and the conservative nano (or conservative micro) data sets is thus the resolution cutoff.

In order to determine whether it is the greater number of images or the smaller beam size that explain the much better statistics of the nano data sets (both conservative and

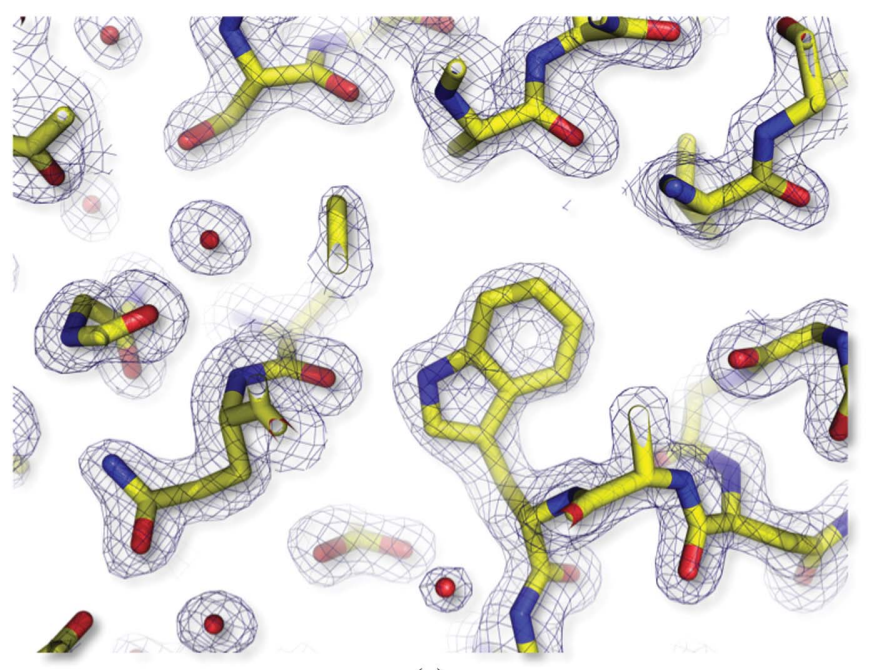

(a)

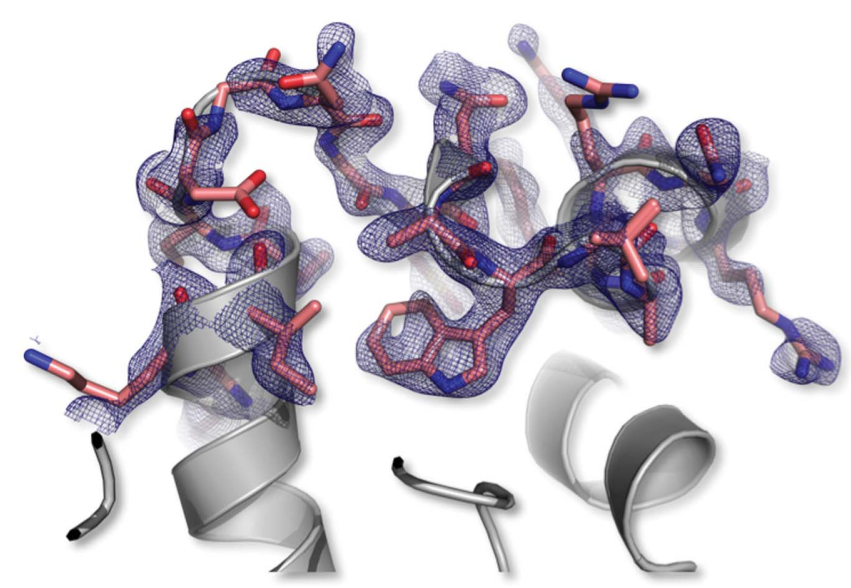

(c)

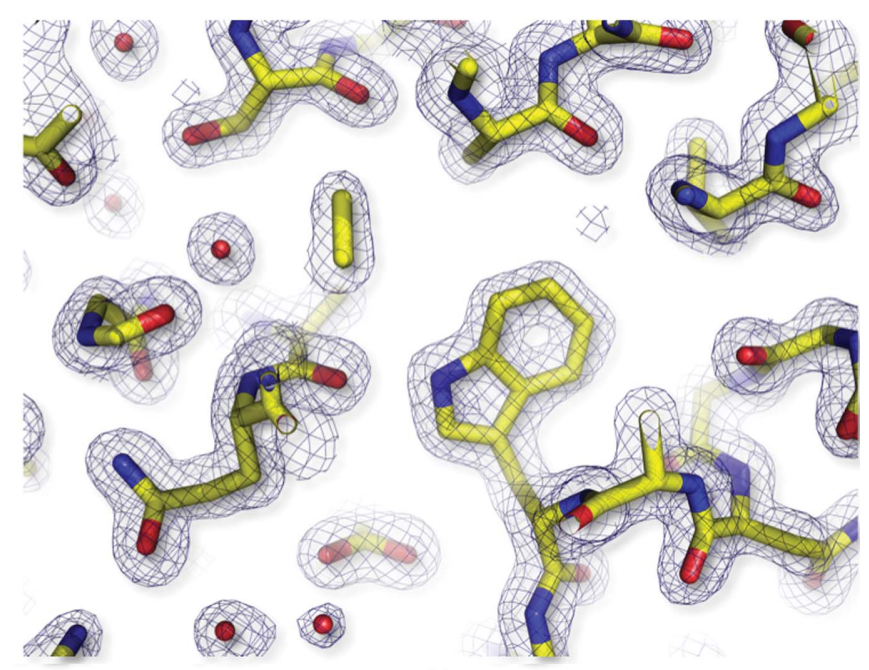

(b)

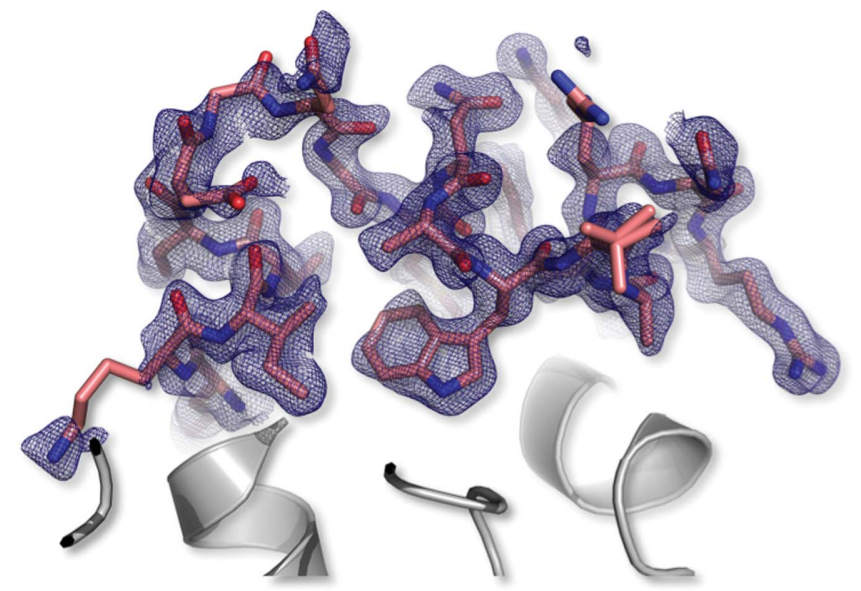

(d)

Figure 4

Unbiased $2 m F_{\mathrm{o}}-D F_{\mathrm{c}}$ OMIT electron-density maps. $(a, b)$ Overview of the electron density around Trp108 in unbiased $2 m F_{\mathrm{o}}-D F_{\mathrm{c}}$ OMIT electrondensity maps (contour level $1.2 \sigma)$ calculated from the progressive micro $(a)$ and progressive nano $(b)$ data sets. $(c, d)$ Unbiased $2 m F_{\mathrm{o}}-D F_{\mathrm{c}}$ electrondensity maps (contour level $1.2 \sigma$ ) calculated from the micro $(c)$ and nano $(d)$ data sets using as a starting model a truncated model of lysozyme lacking residues 96-116. Residues 96-116 are shown as pink sticks, while the residues used for generating the maps are shown as ribbon diagrams. That the electron-density maps shown in $(c)$ and $(d)$ cover virtually all of the atoms in residues $96-116$ highlights the quality of the structural information contained in the progressive micro and progressive nano data sets. 
progressive) when compared with those of the micro data sets, we also generated 'reduced nano' data sets by randomly selecting, in the nano data sets, a number of hits equivalent to that used to produce the micro data set. The same number of indexed patterns was thus used to produce the progressive reduced nano and the conservative reduced nano data sets, which only differ in the resolution cutoff.

\subsection{Structure determination and analysis}

Data were converted to mtz format and phased by molecular replacement using Phaser (McCoy et al., 2007) with PDB entry 1ljn (Harata \& Kenai, 2002) as the starting model. To verify whether the structural information contained in our data sets could have allowed automated model building, we attempted fitting of the full HEWL amino-acid sequence into various molecular-replacement maps using phenix.autobuild with default settings (Terwilliger et al., 2008). The maps were generated from molecular-replacement trials with six different lysozyme models. The first three models consisted of the mainchain and side-chain atoms from residues 1-127 (full protein), 1-65 (N-terminal half) or 66-127 (C-terminal half). The other three were composed of the main-chain atoms of residues 1$127,1-65$ or $66-127$. In all cases, $\sim 98 \%$ of the main chain and $90-98 \%$ of the side chains were reconstructed (Supplementary Table S1).

Structures were refined by iterative cycles of reciprocalspace and real-space refinement. Reciprocal-space refinement was performed using the PHENIX software suite (Adams et al., 2010) and included refinement of coordinates and atomic displacement parameters ( $B$ factors). Manual modifications in real space were performed using Coot (Emsley \& Cowtan, 2004). Unbiased refined $2 m F_{\mathrm{o}}-D F_{\mathrm{c}}$ and $m F_{\mathrm{o}}-D F_{\mathrm{c}}$ electrondensity maps are shown in Figs. $4(a)$ and $4(b)$ for the two progressive data sets. To further assess the quality of these data, residues 96-116 were deleted from the model and reciprocal-space refinement was undertaken (eight cycles of positional and $B$-factor refinement, including two cycles of simulated annealing). In the resulting $2 m F_{\mathrm{o}}-D F_{\mathrm{c}}$ map from both data sets, quasi-perfect electron density is observed around the omitted residues (Figs. $4 c$ and $4 d$ ).

We attempted to compare our data sets (conservative micro and conservative nano) with a variety of previously published HEWL data sets, including (i) two low-dose data sets collected using conventional oscillation methods either at $100 \mathrm{~K}$ (PDB entry 1vds; S. Aibara, A. Suzuki, A. Kidera, K. Shibata, T. Yamane, L. J. DeLucas \& M. Hirose, unpublished work; data collected on a rotating anode) or RT (PDB entry 3zek; in situ data collection on a synchrotron beamline; Pinker et al., 2013), (ii) an SFX data set collected in vacuum and at RT using $40 \mathrm{fs}$ XFEL pulses (PDB entry 4et8; Boutet et al., 2012) and (iii) an SSX data set collected at RT by flowing a crystal slurry across a synchrotron X-ray beam (PDB entry 4o34; Stellato et al., 2014). In the following, these data sets (PDB entries 1vds, $3 z e k, 4$ et8 and 4o34) are referred to as 'low-dose $100 \mathrm{~K}$ ', 'low-dose 295 K', 'SFX' and 'Flow-SSX', respectively. Data sets were scaled using SCALEIT from CCP4 (Winn et al.,
2011; Supplementary Figs. S3 and S4). The unweighted $R_{\text {iso }}$ values between the various data sets are high, suggesting nonisomorphism, whereas the compilation of unit-cell parameters rather points towards all data sets (and the resulting structures) being isomorphous. A possible hypothesis to explain this apparent lack of isomorphism is that it emerges from errors in intensity distributions introduced by the Monte Carlo integration. Accordingly, and as observed previously by Schlichting and coworkers, the weighted $R$ factors are drastically lower (Boutet et al., 2012), in particular at low resolution. However, weighted $R$ factors may not be the most appropriate metric to directly compare data sets collected using different methods, owing to multiple systematic errors in signal-to-noise estimation and scaling. Regardless, our data tend to better agree with the data collected using conventional methods than with the data collected using Flow-SSX or SFX. A possible explanation is that errors introduced during the Monte Carlo integration of the various serial data sets add up during the scaling procedure.

Structure-factor amplitude Fourier difference $\left(F_{\mathrm{o}}-F_{\mathrm{o}}\right)$ maps were calculated between the various data sets after the structure-factor amplitude differences were $q$-weighted to meliorate their estimates (Colletier et al., 2007). $F_{\mathrm{o}}-F_{\mathrm{o}}$ maps between data sets $i$ and $j$ were thus computed using observed structure-factor amplitudes of data sets $i$ and $j$ and calculated phases from model i, i.e. $\left[q w\left(F_{\mathrm{o}}^{j}-F_{\mathrm{o}}^{i}\right)\right] \exp \left(-i \varphi^{j}\right)$. For comparison purposes, all $F_{\mathrm{o}}-F_{\mathrm{o}}$ maps were computed to a resolution of $2.1 \AA$ (i.e. the resolution of the Flow-SSX data set). The figures were produced using PyMOL (Schrödinger), gnuplot or home-made Python scripts.

\section{Results}

\subsection{Data processing}

We used NanoPeakCell (Fig. 2) to identify hits, correct the metrology, subtract background scattering and locate Bragg peaks (Fig. 3). The hit rates were 9.9 and $41.9 \%$ for the micro and nano data sets, respectively (i.e. 6862 and 58647 frames, respectively; Table 2). The drastically lower hit rate attained for the micro data set can be partly explained by the reduced probability of hitting the same crystal twice when the step size of the raster scan is doubled. It is, however, more likely that the crystal concentration in the $\mathrm{Si}_{3} \mathrm{~N}_{4}$ sandwich used to collect the micro data set was sub-optimal.

Indexing and integration were performed using CrystFEL. Data-processing parameters, as well as subtleties specific to serial synchrotron data-processing, are detailed in $\$ 2$. Briefly, 85 and $80 \%$ of NanoPeakCell hits were indexed and integrated for the micro and nano data sets, respectively (Table 2). Reaching such high indexing rates was only possible with background-subtracted data. Merging was performed by Monte Carlo integration (Kirian et al., 2011). For each data set, two integrations were performed using either conservative (1.95 and $1.85 \AA$ resolution cutoffs for the conservative micro and conservative nano data sets, respectively) or progressive $(1.7 \AA$ resolution cutoffs for the progressive micro and progressive nano data sets) criteria. 
Table 3

Comparison of CrystFEL indexing statistics for data collected from lysozyme microcrystals using SFX (Boutet et al., 2012), Flow-SSX (Stellato et al., 2014) and raster-scanning SSX (this work).

Values in parentheses are for the highest resolution shell.

\begin{tabular}{|c|c|c|c|c|c|}
\hline Type of experiment & $\begin{array}{l}\text { SFX, } 40 \text { fs pulses } \\
\text { (Boutet et al., 2012) }\end{array}$ & $\begin{array}{l}\text { SFX, } 5 \text { fs pulses } \\
\text { (Boutet et al., 2012) }\end{array}$ & $\begin{array}{l}\text { Flow-SSX } \\
\text { (Stellato et al., 2014) }\end{array}$ & $\begin{array}{l}\text { Raster-scanning SSX, } \\
\text { conservative micro }\end{array}$ & $\begin{array}{l}\text { Raster-scanning SSX, } \\
\text { conservative nano }\end{array}$ \\
\hline PDB entry & $4 \mathrm{et} 8$ & $4 \mathrm{et} 9$ & 4034 & 4wg1 & $4 w g 7$ \\
\hline Dose per crystal $\dagger$ (MGy) & 33 & 2.9 & 0.3 & 3.2 & 29.1 \\
\hline Dose rate $\left(\mathrm{MGy} \mathrm{s}^{-1}\right)$ & $8.3 \times 10^{14}$ & $5.8 \times 10^{14}$ & 100 & 16 & 291 \\
\hline \multicolumn{6}{|l|}{ Unit-cell parameters $(\AA)$} \\
\hline$a$ & 79.0 & 79.0 & $79.5 \pm 0.3$ & $78.0 \pm 0.3$ & $78.0 \pm 0.2$ \\
\hline$b$ & 79.0 & 79.0 & $79.4 \pm 0.2$ & $78.0 \pm 0.3$ & $78.0 \pm 0.2$ \\
\hline$c$ & 38.0 & 38.0 & $38.4 \pm 0.2$ & $38.3 \pm 0.3$ & $38.5 \pm 0.2$ \\
\hline Upper limit for irradiated volume $\$\left(\mu \mathrm{m}^{3}\right)$ & 3 & 3 & 125 & 75 & 0.5 \\
\hline Resolution $(\AA)$ & $35.90-1.90(2.00-1.90)$ & $35.90-1.90(2.00-1.90)$ & $39.65-2.10(\mathrm{n} / \mathrm{a})$ & $50-1.95(2.05-1.95)$ & $50-1.85(1.95-1.85)$ \\
\hline$\langle I / \sigma(I)\rangle$ & $7.4(2.8)$ & $7.3(3.1)$ & $8.1(1.9)$ & $4.5(2.4)$ & $8.4(2.4)$ \\
\hline$R_{\text {split }} \S$ & $0.158(\mathrm{n} / \mathrm{a})$ & $0.159(\mathrm{n} / \mathrm{a})$ & $0.077(0.540)$ & $0.172(0.390)$ & $0.064(0.375)$ \\
\hline$R_{\text {merge }}(I)$ & $\mathrm{n} / \mathrm{a}$ & $\mathrm{n} / \mathrm{a}$ & $\mathrm{n} / \mathrm{a}$ & $0.238(0.541)$ & $0.090(0.523)$ \\
\hline$R_{\text {merge }}(F)$ & $\mathrm{n} / \mathrm{a}$ & $\mathrm{n} / \mathrm{a}$ & $\mathrm{n} / \mathrm{a}$ & $0.146(0.280)$ & $0.067(0.221)$ \\
\hline $\mathrm{CC}_{1 / 2}-$ & $\mathrm{n} / \mathrm{a}$ & $\mathrm{n} / \mathrm{a}$ & $0.99(0.90)$ & $0.99(0.94)$ & $0.99(0.98)$ \\
\hline Wilson $B \dagger+\left(\AA^{2}\right)$ & 28 & 29 & 44 & 32 & 38 \\
\hline$R_{\text {free }}$ & 0.229 & 0.227 & 0.230 & 0.264 & 0.245 \\
\hline$R_{\text {work }}$ & 0.196 & 0.189 & 0.180 & 0.214 & 0.230 \\
\hline \multicolumn{6}{|l|}{ R.m.s. deviations from ideal values } \\
\hline Bond lengths $(\AA)$ & 0.006 & 0.006 & 0.007 & 0.006 & 0.006 \\
\hline Bond lengths $\left({ }^{\circ}\right)$ & 1.000 & 1.030 & 1.080 & 0.872 & 0.901 \\
\hline No. of frames collected & $1.47 \times 10^{6}$ & $1.99 \times 10^{6}$ & $1.50 \times 10^{6}$ & $0.07 \times 10^{6}$ & $0.14 \times 10^{6}$ \\
\hline Hit rate $(\%)$ & 4.5 & 2.0 & 11.2 & 9.9 & 41.9 \\
\hline No. of indexed frames & 12247 & 10575 & 40233 & 5966 & 35446 \\
\hline Indexing rate $(\%)$ & 18.4 & 26.4 & 24.0 & 86.9 & 79.3 \\
\hline Overall indexing rate $(\%)$ & 0.83 & 0.53 & 2.67 & 8.60 & 33.23 \\
\hline
\end{tabular}

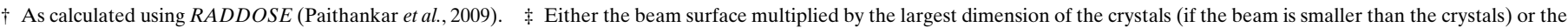

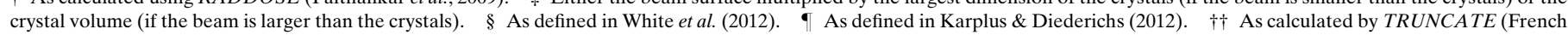
\& Wilson, 1978) in CCP4 (Winn et al., 2011).

\subsection{Quality of the structural information}

All data sets were phased by molecular replacement (MR) using the structure of HEWL in the tetragonal space group (PDB entry 1ljn) as the starting model. The resulting experimental maps were of excellent quality, as shown in Fig. 4 for the progressive micro and progressive nano (1.7 $\AA$ resolution) data sets. To further evaluate the quality of the structural information contained in our data sets, MR phasing was attempted using incomplete starting models. All MR trials succeeded, even when as few as $20 \%$ of the total atoms of HEWL were used. The resulting experimental maps were of sufficient quality to allow the automated building of $\sim 98 \%$ of the main-chain atoms and $90-98 \%$ of the side-chain atoms. Supplementary Table S1 recapitulates these results for the progressive nano data set.

The structures derived from our six data sets (conservative, progressive and progressive reduced micro and conservative, progressive and progressive reduced nano) were refined independently. The final statistics of the conservative micro and nano structures are of comparable quality (Table 2), and likewise for the progressive micro and nano structures. To decide on the effective resolution cutoffs to apply to the progressive micro and progressive nano data sets, we performed iterative pair refinement as described in Karplus \& Diederichs (2012). For both progressive data sets, we observed a decrease in the $R_{\text {free }}$ value at resolution $A$ when the model was refined including structural information up to a higher resolution $B$. This demonstrates that in both data sets valid structural information is present beyond the conservative resolution cutoff (1.95 and $1.85 \AA$, respectively; Supplementary Fig. S2). The $\mathrm{CC}_{1 / 2}$ values in the highest resolution shell (1.78-1.70 $\AA$ ) are 0.66 and 0.85 for the progressive micro and progressive nano data sets, respectively. The corresponding $\langle I / \sigma(I)\rangle$ values drop below 1 ( 0.64 and 0.87 for the progressive micro and progressive nano data sets, respectively) and the $R_{\text {split }}$ values rise above $100 \%$. For the sake of completeness and consistency we have deposited four models in the PDB (Table 2) corresponding to the progressive and conservative integrations of the micro and nano data sets, respectively.

\subsection{Radiation damage}

It has been shown that beyond the limit of 29 MGy, the biological information obtained from a protein crystal at $100 \mathrm{~K}$ is compromised (Owen et al., 2006). At RT, the extent of radiation damage is increased by $\sim 100$-fold (Nave \& Garman, 2005), suggesting a dose limit of $0.3 \mathrm{MGy}$. Here, the doses per diffraction pattern, and hence per data set, are 3.2 and 29.1 MGy for the micro and nano data sets, respectively. It was therefore expected that strong radiation damage would occur, notably at highly radiation-sensitive sites such as disulfide bridges. Accordingly, we observed, in structure-factor amplitude Fourier difference $\left(F_{\mathrm{o}}-F_{\mathrm{o}}\right)$ maps calculated between our data sets and low-dose data sets collected at both $100 \mathrm{~K}$ (low-dose $100 \mathrm{~K}$ ) and RT (low-dose $295 \mathrm{~K}$ ), strong negative 
peaks $(-4 \sigma)$ on the $\mathrm{S}$ atoms of HEWL, and most particularly, on disulfide bridges (Fig. 5a). Interestingly, we observed that the disulfide bridge Cys64-Cys80 is the most radiationsensitive at RT, whereas it is Cys30-Cys115 that is the most sensitive at $100 \mathrm{~K}$ (Sutton et al., 2013). Specific damage was, however, unapparent in $2 m F_{\mathrm{o}}-D F_{\mathrm{c}}$ and $m F_{\mathrm{o}}-D F_{\mathrm{c}}$ maps, indicating that disulfide bridges are not broken in the micro and the nano data sets (Fig. $5 b$ ). It has been shown that upon X-ray damage disulfide bridges contract (Sutton et al., 2013) and/or elongate (Carpentier et al., 2010; Weik et al., 2002)

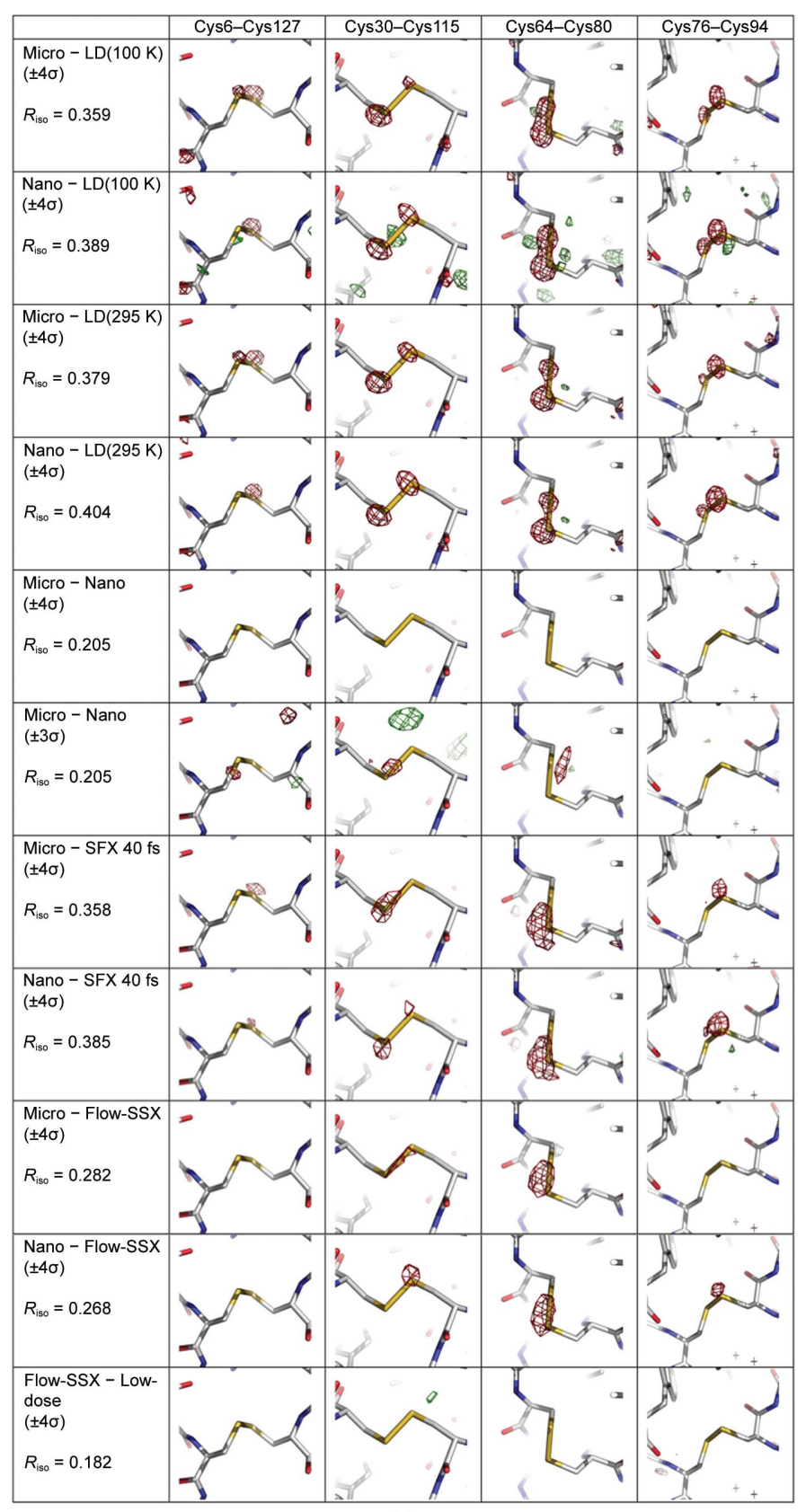

(a) before breaking. It may thus be that in our structures disulfide bridges are trapped in one of these intermediate stages. Regardless, Fig. 5(b) indicates that the extent of radiation damage is limited in our data sets and that the structural information has thus not yet been compromised.

The approximately tenfold higher flux density of the nanofocused beam was suggestive of possible higher radiation damage in the nano data set compared with the micro data set. Surprisingly, however, the $F_{\mathrm{o}}^{\text {micro }}-F_{\mathrm{o}}^{\text {nano }}$ map did not indicate stronger damage to disulfide bridges in the nano structure. Rather, it revealed a small $(-3 \sigma)$ negative peak at the most sensitive disulfide bridge at RT (Cys64-Cys80), suggesting that the micro data set is more damaged than the nano data set. A possible rationale for this observation is thus that for the order-of-magnitude higher dose rate of the nano data set $\left(291 \mathrm{MGy} \mathrm{s}^{-1}\right.$ ) a lag phase occurs that delays the appearance of specific radiation damage. Additional experiments will be required in order to confirm or discard this hypothesis.

We also endeavoured to compare our data with those produced by other serial crystallography approaches (SFX and Flow-SSX; see \$2) on the same system (HEWL) and at the same temperature (RT). Table 3 shows that the three structures are roughly comparable in terms of $R_{\text {split }}(0.158,0.077$ and 0.064 for the SFX, Flow-SSX and conservative nano data sets, respectively), $\langle I / \sigma(I)\rangle$ (7.4, 8.1 and 8.4, respectively), $\mathrm{CC}_{1 / 2}$ (not given for the SFX data set; 0.99 and 0.99 for the Flow-SSX and conservative nano data sets, respectively), resolution $\left(1.9,2.1\right.$ and $1.85 \AA$, respectively), $R_{\text {work }}(0.196$, 0.180 and 0.230 , respectively) and $R_{\text {free }}(0.229,0.230$ and 0.245 , respectively). Yet, the dose and dose rate were $33 \mathrm{MGy}$ and $8.3 \times 10^{14} \mathrm{MGy} \mathrm{s}^{-1}$, respectively, for the SFX data set (Boutet et al., 2012) and $0.3 \mathrm{MGy}$ and $100 \mathrm{MGy} \mathrm{s}^{-1}$, respectively, for the Flow-SSX data set (Stellato et al., 2014). Thus, the FlowSSX data set is comparable to the nano data set in terms of dose rate (100 versus $291 \mathrm{MGy} \mathrm{s}^{-1}$ ) but not in terms of dose ( 0.3 versus $29.1 \mathrm{MGy}$ ). The SFX data set is comparable to the nano data set in terms of dose ( 33 versus $29.1 \mathrm{MG} \mathrm{s}^{-1}$ ) but not in terms of dose rate $\left(8.3 \times 10^{14}\right.$ versus $\left.291 \mathrm{MGy} \mathrm{s}^{-1}\right)$. Nevertheless, radiation damage was clearly visible in both the $F_{\mathrm{o}}^{\mathrm{SFX}}-F_{\mathrm{o}}^{\text {nano }}$ and the $F_{\mathrm{o}}^{\text {Flow-SSX }}-F_{\mathrm{o}}^{\text {nano }} F$ Fourier difference maps (Fig. 5a). This result was expected; the shortness of XFEL pulses indeed allows diffraction to occur before

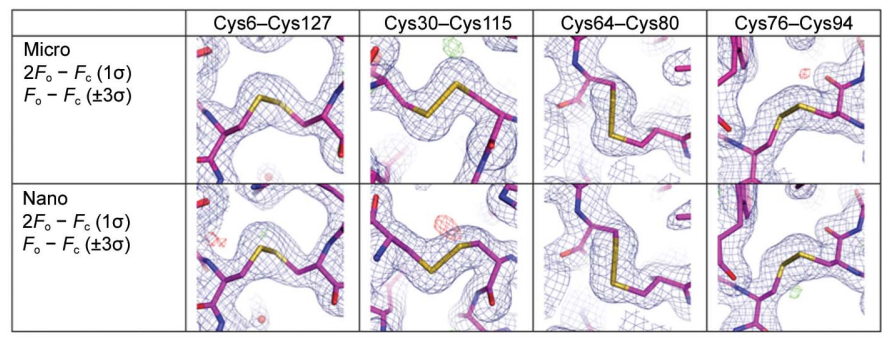

$(b)$

Figure 5

Specific radiation damage is observed in both the micro and the nano data sets, but does not compromise the structural information. (a) Structure-factor amplitude Fourier difference $\left(F_{\mathrm{o}}-F_{\mathrm{o}}\right)$ maps were calculated between our data sets (conservative micro and conservative nano) and four other HEWL data sets: the SFX data set, the Flow-SSX data set and two low-dose data sets collected at either 100 or $295 \mathrm{~K}$. The maps are displayed around the four disulfide bridges of lysozyme, with red and green contours indicating negative and positive density, respectively. $(b)$ Unbiased $2 m F_{\mathrm{o}}-D F_{\mathrm{c}}($ blue; contour level $1.0 \sigma$ ) and $m F_{\mathrm{o}}-D F_{\mathrm{c}}$ (red and green; contour level $\pm 3 \sigma$ ) maps are displayed around the four disulfide bridges of lysozyme. 
radiation damage can take place, whereas the reduced doses, together with a relatively high dose rate, have allowed mitigation of the appearance of specific damage in the Flow-SSX study. In conclusion, the three approaches yield data of comparable quality, suggesting that our raster-scanning approach could be a useful alternative to collect serial crystallography data from, for example, scarce crystalline samples. That our data sets are more radiation-damaged than those from SFX and Flow-SSX is acknowledged, yet this does not seem to significantly alter the quality of the structural information.

\section{Discussion}

We have reported a new approach for the collection of highresolution structural data from macromolecular crystals at RT using raster-scanning serial data collection. Two data sets were collected from tetragonal HEWL crystals on the ID13 beamline at the ESRF, using either a micro-focused (ID13-EH2) or a nano-focused (ID13-EH3) beam. Crystals were presented to the X-ray beam in an $\mathrm{Si}_{3} \mathrm{~N}_{4}$ sandwich at RT (Fig. 1). During the whole experiment, including controls, $1 \mathrm{mg}$ of crystalline sample was consumed.

In order to detect hits and correct for both distortion of the detector and background scattering (Fig. 3), we developed the Python-based software NanoPeakCell (Fig. 2) that performs these tasks in a timely (multi-processing) and user-friendly (graphical user interface) fashion. NanoPeakCell uses the FabIO library (Knudsen et al., 2013) and is therefore able to process data from a variety of detector types including ADSC, MAR CCD and Pilatus detectors. It can also read HDF5, SACLA-HDF5 and LCLS-XTC files and can thus be used to process XFEL data (details to be published elsewhere). NanoPeakCell can output data in EDF (European Data Format), HDF5 (suitable for processing by CrystFEL) and/or 'pickle' (suitable for processing by cctbx.xfel) formats. In the latter case, the data are automatically padded so that the beam centre matches the centre of the image, as currently required for processing data with cctbx.xfel. NanoPeakCell can also generate input files for CrystFEL (geometry and beam description) and calculates a maximum projection of the corrected data on the fly (Supplementary Figs. S1c and S1d). Finally, NanoPeakCell can perform a Bragg-peak search on corrected images (Figs. 2 and 3); the resulting peak list is either included in a supplementary data set of the HDF5 file or printed in a text file. The NanoPeakCell peak list can be used as a guide for adjusting CrystFEL and/or cctbx.xfel spotfinding parameters, or directly fed into CrystFEL for indexing. In indexing tests performed using either the peak-finding algorithm implemented in CrystFEL or that implemented in NanoPeakCell, similar indexing rates were obtained (not shown). While peak searching is faster in CrystFEL than in NanoPeakCell, the computing cost is minor if NanoPeakCell is used to perform hit finding and data correction.

Indexing, integration and merging were performed using CrystFEL (Table 2). Good statistics were obtained for both the micro and the nano data sets, demonstrating that raster- scanning SSX using either micro- or nano-focused beams is a promising approach in RT macromolecular X-ray crystallography. The micro data set furthermore sustains that $\sim 6000$ indexed hits are sufficient to obtain a reasonably good structure, at least when a molecular-replacement model is available (Figs. $4 a$ and $4 c$, Table 2). Of note, background subtraction was compulsory to produce data sets of such quality.

As judged from statistical indicators such as $\langle I / \sigma(I)\rangle, \mathrm{CC}_{1 / 2}$ and $R_{\text {split }}$ for the raw data, and $R_{\text {free }}$ and $R_{\text {work }}$ for the refined structures, the data obtained using raster-scanning SSX are of comparable quality to those produced by SFX and Flow-SSX (Table 3). The fairest comparison is between the Flow-SSX and the nano data sets as both were produced using a similar number of images ( 40 000 versus $\sim 46000$ indexed frames for the Flow-SSX and nano data sets, respectively) collected on a similar X-ray source. Differences between the two approaches are the crystal presentation procedure (crystal stream in Flow-SSX versus immobilized crystals on a solid support in the present study), the beam size $(6 \times 9 \mu \mathrm{m}$ versus $0.15 \times 0.175 \mu \mathrm{m})$, the dose per crystal $(0.3$ versus $29.1 \mathrm{MGy})$ and the dose rate (100 versus $291 \mathrm{MGy} \mathrm{s}^{-1}$ ). Of course, higher $R_{\text {split }}$ and lower $\langle I / \sigma(I)\rangle$ values are observed in the highest resolution shells of the progressive nano data set (Tables 2 and 3), given that we intentionally applied less stringent resolution cutoffs in our progressive integrations. Indeed, we have relied on the observation of signal out to the edge of the detector in the maximum projections from indexed patterns (Supplementary Fig. S1) and on subsequently performed iterative pair refinement analyses (Supplementary Fig. 2) to determine resolution cutoffs for the progressive data sets. Statistics produced by the raster-scanning and the Flow-SSX approaches are nevertheless of similar quality when the high-resolution limit criteria are the same, i.e. $\langle I / \sigma(I)\rangle>2$ (1.9 and 2.37 for the Flow-SSX and conservative nano data sets, respectively) and $R_{\text {split }} \leq \sim 50 \%$ (54.0 and $37.5 \%$ for the Flow-SSX and conservative nano data sets, respectively). Of note, the conservative nano data set extends to $1.85 \AA$ resolution compared with $2.1 \AA$ resolution for the Flow-SSX data set. Yet, our conservative nano data set suffers from a lower redundancy of 787 (430 in the highest resolution shell) compared with 1755 (1281) for the Flow-SSX data set. At least three factors could be contributing to this: the radius of the three-dimensional intensity profile used for the CrystFEL integration $\left(5 \times 10^{-4} \AA^{-1}\right.$ in the present study; not available for the Flow-SSX data set), the beam divergence ( 0.3 and $1.0 \mathrm{mrad}$, respectively) and the fact that in the flow approach the crystals do not remain still during exposure but sometimes roll, thus giving rise to pseudo-oscillation.

Regardless, it is clear that our data sets are more radiationdamaged than those produced by SFX (Boutet et al., 2012) and Flow-SSX (Stellato et al., 2014) (Fig. 5a). Indeed, in an attempt to compensate for the lower flux and the relatively small irradiated volume at the nano-focused beamline (upper limit of $0.5 \mu \mathrm{m}^{3}$ ), the nano data set was collected using a dose per image (29.1 MGy) just below the experimental dose limit defined by Garman and coworkers (30 MGy; Owen et al., 2006) beyond which the cumulative information collected 
from a macromolecular crystal at $100 \mathrm{~K}$ is compromised. The micro data set was collected using a dose that was approximately ten times lower, i.e. 3.2 MGy. Given that radiation damage proceeds $\sim 100$ times faster at RT than at $100 \mathrm{~K}$ (Nave \& Garman, 2005), the theoretical estimate for the dose limit at RT is $0.3 \mathrm{MGy}$, implying that the micro and nano data sets are overdosed by factors of 10 and 100, respectively. Accordingly, specific damage was observed to disulfide bridges in the $F_{\mathrm{o}}-F_{\mathrm{o}}$ maps calculated between our data sets and low-dose data sets collected either on a rotating anode at $100 \mathrm{~K}$ or in situ at RT (Fig. 5a). Specific damage was also visible in $F_{\mathrm{o}}-F_{\mathrm{o}}$ maps calculated between our data sets and either the SFX or the Flow-SSX data sets (Fig. $5 a$ ).

Yet, no sign of specific damage was visible in the unbiased $2 m F_{\mathrm{o}}-D F_{\mathrm{c}}$ and $m F_{\mathrm{o}}-D F_{\mathrm{c}}$ maps calculated from both the micro and the nano data sets. More importantly, the structural information proved to be of sufficient quality to allow MR phasing with partial search models (down to $20 \%$ of the total atoms) and quasi-complete main-chain (98\%) and side-chain (90-98\%) fitting in the resulting maps of the HEWL sequence (Supplementary Table S1). A possible explanation for the limited damage observed in our data, and in the nano data set in particular, is that crystals suffer less damage when impinged with X-rays a single time (no cumulative effect) and at a very high dose rate (16 and $291 \mathrm{MGy} \mathrm{s}^{-1}$ for the micro and nano data sets, respectively). It has already been shown that data collection at high dose rates delays the appearance of radiation damage at RT (Owen et al., 2014; Southworth-Davies et al., 2007). Admittedly, the highest dose rate used in the study by Owen et al. (2014) was $25 \mathrm{MGy} \mathrm{s}^{-1}$ and the lag phase lasted about $500 \mathrm{kGy}$ for all proteins, whereas in our experiments $500 \mathrm{kGy}$ would have been delivered in $1.7 \mathrm{~ms}$ for the nano data set. Thus, the $500 \mathrm{kGy}$ lag phase would have expired well within the exposure time for each image ( $0.1 \mathrm{~s})$. It may thus be that for the magnitude-higher dose rate of the nano data set $\left(291 \mathrm{MGy} \mathrm{s}^{-1}\right.$ ) a further lag phase occurs. That the micro data set $\left(16 \mathrm{MGy} \mathrm{s}^{-1}\right)$ appears to be more damaged than the nano data set supports this hypothesis (Fig. 5a). Again, additional experiments are required to confirm or discard this hypothesis.

A variety of sample-presentation strategies exist that either have been used or are amenable to SSX. These include flowing crystals across the X-ray beam by means of a capillary (Stellato et al., 2014), a liquid jet (DePonte et al., 2009; Sierra et al., 2012) or a lipid cubic phase injector (Weierstall et al., 2014) or ejecting them in droplets using acoustic waves (Soares et al., 2011). Solid supports have also been used either to collect oscillation data (Zarrine-Afsar et al., 2012) or still shots (Gebhardt et al., 2014; Hunter et al., 2014). Here, we used a $\mathrm{Si}_{3} \mathrm{~N}_{4}$ membrane sandwich to present the crystals to the X-ray beam. This approach has advantages, including the small amount of micro-crystalline sample required $(1 \mathrm{mg}$ of protein compared with $250 \mathrm{mg}$ in the study by Stellato et al., 2014), an adjustable hit rate ( $\sim 10$ and $40 \%$ in the micro and nano data sets, respectively), a high indexing rate ( 85 and $80 \%$ in the micro and nano data sets, respectively) and a minimized background scattering. Also, the proposed mounting procedure is virtually manipulation-free, suggesting that it could be used on fragile micro-crystals. Data collection at RT is another advantage, given the inherently flexible nature of biological material. Of course, this is a superiority to oscillation data collection at cryogenic temperature which raster-scanning SSX shares with the other serial crystallography approaches. It is tantalizing to propose that raster-scanning SSX could be utilized in kinetic crystallography experiments, in which a light source is used to 'pump' a macromolecular crystalline system (either a light-driven protein or a complex of a light-insensitive protein with a photocleavable caged compound) and the $\mathrm{X}$-ray beam is used to 'probe' the resulting structure (Bourgeois \& Royant, 2005; Colletier et al., 2007). The attainable time resolution would essentially be limited by the length of the X-ray pulse and the detector readout, thus allowing timeresolved structural studies on the microsecond to second timescale, i.e. that pertinent to protein kinetics. X-rays could also be used as both the pump and the probe in the framework of 'shoot-and-trap' experiments (Colletier et al., 2008) or to structurally monitor the appearance and evolution of radiation damage at RT in an oscillation-free, and thus damageaccumulation-free, fashion. The observation that the Cys64Cys 80 disulfide bridge is the most radiation-sensitive at RT (Fig. 5a), instead of Cys30-Cys115 as at $100 \mathrm{~K}$ (Sutton et al., 2013), advocates for the specific investigation of macromolecular radiation-sensitivity at RT.

A current weakness of SSX is the time that is required to collect a full data set. In the study by Stellato et al. (2014), $17 \mathrm{~h}$ were needed to produce a data set of 40000 useable frames, owing to a comparatively low overall success rate. Although our success rates are higher overall, the nano data set consisting of 46000 useable frames required $19 \mathrm{~h}$ to be produced owing to the comparatively slow readout time of our Frelon CCD detector. The ID13 beamline at the ESRF is now equipped with a new Dectris 4M Eiger detector, which will allow a drastic increase in the data-collection rate $(1.3 \mathrm{~ms}$ sampling rate, $6 \mu$ s readout time). Also, Phase II of the ESRF upgrade, which involves a substantial upgrade of the storage ring, will lead to an increase of about a factor of 50 in brilliance. Based on this and other improvements, a 10000 -fold increase in the flux of the nano-beam can be expected, which will dramatically reduce the minimum exposure time required to collect high-resolution data.

\section{Acknowledgements}

Financial support by the ANR, the CEA, the CNRS and the UJF is acknowledged. NC is supported by a grant from the France Alzheimer Foundation. We thank M. Weik, T. A. White, R. G. Sierra, C. Riekel, J. Rodriguez, E. Girard, D. Cascio, M. R. Sawaya and D. S. Eisenberg for helpful and stimulating discussions, as well as for continuing support. We are indebted to L. Lardière and L. Peyrin for the threedimensional rendering of our sample preparation procedure shown in Fig. 1(f).

\section{References}

Adams, P. D. et al. (2010). Acta Cryst. D66, 213-221. 
Bourgeois, D. \& Royant, A. (2005). Curr. Opin. Struct. Biol. 15, 538-547.

Boutet, S. et al. (2012). Science, 337, 362-364.

Carpentier, P., Royant, A., Weik, M. \& Bourgeois, D. (2010). Structure, 18, 1410-1419.

Chapman, H. N. et al. (2011). Nature (London), 470, 73-77.

Chen, V. B., Arendall, W. B., Headd, J. J., Keedy, D. A., Immormino, R. M., Kapral, G. J., Murray, L. W., Richardson, J. S. \& Richardson, D. C. (2010). Acta Cryst. D66, 12-21.

Coan, P., Peterzol, A., Fiedler, S., Ponchut, C., Labiche, J. C. \& Bravin, A. (2006). J. Synchrotron Rad. 13, 260-270.

Cohen, A. E. et al. (2014). Proc. Natl Acad. Sci. USA, 111, 1712217127.

Cole, A. (1969). Radiat. Res. 38, 7-33.

Colletier, J.-P., Bourgeois, D., Sanson, B., Fournier, D., Sussman, J. L., Silman, I. \& Weik, M. (2008). Proc. Natl Acad. Sci. USA, 105, 11742-11747.

Colletier, J.-P., Royant, A., Specht, A., Sanson, B., Nachon, F., Masson, P., Zaccai, G., Sussman, J. L., Goeldner, M., Silman, I., Bourgeois, D. \& Weik, M. (2007). Acta Cryst. D63, 1115-1128.

DePonte, D. P., Doak, R. B., Hunter, M., Liu, Z., Weierstall, U. \& Spence, J. C. (2009). Micron, 40, 507-509.

Duisenberg, A. J. M. (1992). J. Appl. Cryst. 25, 92-96.

Emsley, P. \& Cowtan, K. (2004). Acta Cryst. D60, 2126-2132.

Engh, R. A. \& Huber, R. (1991). Acta Cryst. A47, 392-400.

Fraser, J. S., van den Bedem, H., Samelson, A. J., Lang, P. T., Holton, J. M., Echols, N. \& Alber, T. (2011). Proc. Natl Acad. Sci. USA, 108, 16247-16252.

French, S. \& Wilson, K. (1978). Acta Cryst. A34, 517-525.

Gati, C., Bourenkov, G., Klinge, M., Rehders, D., Stellato, F., Oberthür, D., Yefanov, O., Sommer, B. P., Mogk, S., Duszenko, M., Betzel, C., Schneider, T. R., Chapman, H. N. \& Redecke, L. (2014). IUCrJ, 1, 87-94.

Gebhardt, R., Teulon, J.-M., Pellequer, J.-L., Burghammer, M., Colletier, J.-P. \& Riekel, C. (2014). Soft Matter, 10, 5458-5462.

Harata, K. \& Kenai, R. (2002). Proteins, 48, 53-62.

Hunter, M. S. et al. (2014). Sci. Rep. 4, 6026.

Kabsch, W. (2010). Acta Cryst. D66, 125-132.

Karplus, P. A. \& Diederichs, K. (2012). Science, 336, 1030-1033.

Kirian, R. A., White, T. A., Holton, J. M., Chapman, H. N., Fromme, P., Barty, A., Lomb, L., Aquila, A., Maia, F. R. N. C., Martin, A. V., Fromme, R., Wang, X., Hunter, M. S., Schmidt, K. E. \& Spence, J. C. H. (2011). Acta Cryst. A67, 131-140.

Knudsen, E. B., Sørensen, H. O., Wright, J. P., Goret, G. \& Kieffer, J. (2013). J. Appl. Cryst. 46, 537-539.

Koopmann, R. et al. (2012). Nature Methods, 9, 259-262.

Lomb, L. et al. (2011). Phys. Rev. B, 84, 214111.

McCoy, A. J., Grosse-Kunstleve, R. W., Adams, P. D., Winn, M. D., Storoni, L. C. \& Read, R. J. (2007). J. Appl. Cryst. 40, 658674.

Motlagh, H. N., Wrabl, J. O., Li, J. \& Hilser, V. J. (2014). Nature (London), 508, 331-339.

Nave, C. \& Garman, E. F. (2005). J. Synchrotron Rad. 12, 257260.
Neutze, R. (2014). Philos. Trans. R. Soc. Lond. B Biol. Sci. 369, 20130318.

Neutze, R., Wouts, R., van der Spoel, D., Weckert, E. \& Hajdu, J. (2000). Nature (London), 406, 752-757.

Owen, R. L., Paterson, N., Axford, D., Aishima, J., Schulze-Briese, C., Ren, J., Fry, E. E., Stuart, D. I. \& Evans, G. (2014). Acta Cryst. D70, 1248-1256.

Owen, R. L., Rudiño-Piñera, E. \& Garman, E. F. (2006). Proc. Natl Acad. Sci. USA, 103, 4912-4917.

Paithankar, K. S., Owen, R. L. \& Garman, E. F. (2009). J. Synchrotron Rad. 16, 152-162.

Pinker, F., Brun, M., Morin, P., Deman, A.-L., Chateaux, J.-F., Oliéric, V., Stirnimann, C., Lorber, B., Terrier, N., Ferrigno, R. \& Sauter, C. (2013). Cryst. Growth Des. 13, 3333-3340.

Powell, H. R., Johnson, O. \& Leslie, A. G. W. (2013). Acta Cryst. D69, 1195-1203.

Redecke, L. et al. (2013). Science, 339, 227-230.

Sauter, N. K., Hattne, J., Grosse-Kunstleve, R. W. \& Echols, N. (2013). Acta Cryst. D69, 1274-1282.

Sawaya, M. R. et al. (2014). Proc. Natl Acad. Sci. USA, 111, 1276912774.

Schroer, C. G., Kurapova, O., Patommel, J., Boye, P., Feldkamp, J., Lengeler, B., Burghammer, M., Riekel, C., Vincze, L., van der Hart, A. \& Küchler, M. (2005). Appl. Phys. Lett. 87, 124103.

Sierra, R. G. et al. (2012). Acta Cryst. D68, 1584-1587.

Soares, A. S., Engel, M. A., Stearns, R., Datwani, S., Olechno, J., Ellson, R., Skinner, J. M., Allaire, M. \& Orville, A. M. (2011). Biochemistry, 50, 4399-4401.

Southworth-Davies, R. J., Medina, M. A., Carmichael, I. \& Garman, E. F. (2007). Structure, 15, 1531-1541.

Stellato, F. et al. (2014). IUCrJ, 1, 204-212.

Stevenson, H. P., DePonte, D. P., Makhov, A. M., Conway, J. F., Zeldin, O. B., Boutet, S., Calero, G. \& Cohen, A. E. (2014). Philos. Trans. R. Soc. Lond. B Biol. Sci. 369, 20130322.

Sugahara, M. et al. (2015). Nature Methods, 12, 61-63.

Sutton, K. A., Black, P. J., Mercer, K. R., Garman, E. F., Owen, R. L., Snell, E. H. \& Bernhard, W. A. (2013). Acta Cryst. D69, 2381-2394.

Terwilliger, T. C., Grosse-Kunstleve, R. W., Afonine, P. V., Moriarty, N. W., Zwart, P. H., Hung, L.-W., Read, R. J. \& Adams, P. D. (2008). Acta Cryst. D64, 61-69.

Weierstall, U. et al. (2014). Nature Commun. 5, 3309.

Weik, M. \& Colletier, J.-P. (2010). Acta Cryst. D66, 437-446.

Weik, M., Bergès, J., Raves, M. L., Gros, P., McSweeney, S., Silman, I., Sussman, J. L., Houée-Levin, C. \& Ravelli, R. B. G. (2002). J. Synchrotron Rad. 9, 342-346.

White, T. A., Barty, A., Stellato, F., Holton, J. M., Kirian, R. A., Zatsepin, N. A. \& Chapman, H. N. (2013). Acta Cryst. D69, 12311240.

White, T. A., Kirian, R. A., Martin, A. V., Aquila, A., Nass, K., Barty, A. \& Chapman, H. N. (2012). J. Appl. Cryst. 45, 335-341.

Winn, M. D. et al. (2011). Acta Cryst. D67, 235-242.

Zarrine-Afsar, A., Barends, T. R. M., Müller, C., Fuchs, M. R., Lomb, L., Schlichting, I. \& Miller, R. J. D. (2012). Acta Cryst. D68, 321-323. 